\title{
Three-dimensional analysis of wakefields generated by flat electron beams in planar dielectric-loaded structures
}

\author{
D. Mihalcea, ${ }^{1}$ P. Piot, ${ }^{1,2}$ and P. Stoltz ${ }^{3}$ \\ ${ }^{1}$ Northern Illinois Center for Accelerator \& Detector Development and Department of Physics, Northern Illinois University, \\ DeKalb, Illinois 60115, USA \\ ${ }^{2}$ Accelerator Physics Center, Fermi National Accelerator Laboratory, Batavia, Illinois 60510, USA \\ ${ }^{3}$ Tech-X Corporation, Boulder, Colorado 80303, USA \\ (Received 30 April 2012; published 7 August 2012)
}

\begin{abstract}
An electron bunch passing through a dielectric-lined waveguide generates Čerenkov radiation that can result in a high-peak axial electric field suitable for acceleration of a subsequent bunch. Axial fields beyond gigavolt-per-meter are attainable in structures with sub-mm sizes depending on the achievement of suitable electron bunch parameters. A promising configuration consists of using a planar dielectric structure driven by flat electron bunches. In this paper we present a three-dimensional analysis of wakefields produced by flat beams in planar dielectric structures thereby extending the work of Tremaine, Rosenzweig, and Schoessow, Phys. Rev. E 56, 7204 (1997)] on the topic. We especially provide closed-form expressions for the normal frequencies and field amplitudes of the excited modes and benchmark these analytical results with finite-difference time-domain particle-in-cell numerical simulations. Finally, we implement a semianalytical algorithm into a popular particle-tracking program thereby enabling start-to-end high-fidelity modeling of linear accelerators based on dielectric-lined planar waveguides.
\end{abstract}

DOI: 10.1103/PhysRevSTAB.15.081304

PACS numbers: 29.27.- a, 41.20.Jb, 41.60.Bq

\section{INTRODUCTION}

Next generation multi-TeV high-energy-physics lepton accelerators are likely to be based on nonconventional acceleration techniques given the limitations of radiofrequency (rf) normal-conducting [1] and superconducting [2] structures. Nonconventional approaches based on the laser plasma-wakefield accelerator have recently demonstrated average energy gradients one order of magnitude higher than those possible with state-of-the art conventional structures [3]. The applicability of laser-driven techniques to high-energy accelerators is currently limited as attaining luminosity values similar to those desired at the International Linear Collider would demand a laser with power approximately 4 orders of magnitude larger than the most powerful lasers currently available [4]. Another class of nonconventional accelerating techniques includes beam-driven methods which rely on using wakefields produced by high-charge drive bunches traversing a highimpedance structure to accelerate subsequent witness bunches [5]. Such an approach has the advantage of circumventing the use of an external power source and can therefore operate at $\mathrm{mm}$ and sub-mm wavelengths. Structures capable of supporting wakefield generation include plasmas [6] and dielectric-loaded waveguides [7].

Published by the American Physical Society under the terms of the Creative Commons Attribution 3.0 License. Further distribution of this work must maintain attribution to the author(s) and the published article's title, journal citation, and DOI.
The possible use of plasma-wakefield accelerators as the backbone of a multi-TeV electron-positron linear collider is limited by plasma ions motion due to the intense electromagnetic field of the bunch [8]. Dielectric wakefield accelerators (DWFAs) are not prone to similar limitations.

In this paper we concentrate on the collinear DWFA. In such a configuration a highly charged drive bunch propagates through a dielectric-lined waveguide (DLW) and excites an electromagnetic wake $[7,9,10]$. A delayed witness bunch moving on the same path as the drive bunch can experience an accelerating field.

Recent experiments [11] confirm that DLW can support accelerating fields in excess of a $\mathrm{GV} / \mathrm{m}$ thereby making DWFA a plausible candidate for the next-generation highenergy-physics linear accelerators [12] or compact shortwavelength free-electron lasers [13].

In cylindrically symmetric DLW structures, the electric field amplitude of the wakefield is approximately inverse proportional to the aperture radius. Given the linear charge scaling of the field, peak electric field amplitude of the order of gigavolt-per-meter can be obtained by either using high-charge drive beams $(>100 \mathrm{nC})$ in mm-sized DLW structures or by focusing sub-nC bunches in micron-sized DLW structures $[10,14,15]$.

To date, cylindrically symmetric DLW's have been extensively studied both theoretically $[10,16-18]$ and experimentally [19-22]. Since the angular divergence of the beam also increases during focusing it is hard to maintain a low transverse size of the beam over a long propagation distance. Therefore, the design of the DLW structures must 
compromise between a small transverse size to maximize the intensity of the wakefield, and a longer interaction length to maximize the energy gain of the test charge. An appealing solution consists of using flat electron beams passing through slab-geometry DLWs [14,23-25]. This possibility has become more attractive since the recent advances toward generating flat beams directly in photoinjectors [26,27].

In the following sections we present an analysis of the generation of wakefields in rectangular DLW structures excited by drive beams with arbitrary three-dimensional charge distributions. The main goal of this paper is to extend the formalism introduced in Ref. [14] by including all types of modes excited in a planar DLW. This paper is pedagogical in the sense that it is about the method of derivation rather than the results, which have been derived in previous papers, most extensively in Ref. [24]. However, this paper provides close-form formulas for the eigenfrequencies and electromagnetic fields excited in planar DLWs. These analytical results are benchmarked against three-dimensional finite-difference timedomain (FDTD) simulations. Finally, the model is implemented in a popular particle-in-cell (PIC) beam dynamics program. The latter provides a fast and highfidelity model enabling start-to-end simulation of DLWbased linear accelerators.

\section{WAKEFIELD GENERATION}

The geometry of the problem analyzed in this paper is depicted in Fig. 1. Transient effects resulting from the injection of the electron bunch in the structure are not included (the structure is assumed to be infinitely long) and the drive bunch is taken to be ultrarelativistic with its Lorentz factor $\gamma$ much greater than unity. The building block of a real drive bunch is assumed to consist of a finitewidth linear charge distribution oriented along the horizontal $x$ axis which moves in the $z$ direction with velocity $v$ and has offset $y_{0}$ in the vertical direction. To simplify, it is also assumed that the linear charge distribution is symmetric in the $x$ coordinate and it vanishes at the sides of the dielectric structure. Under these assumptions the charge distribution can be written as a Fourier series in the horizontal direction [14],

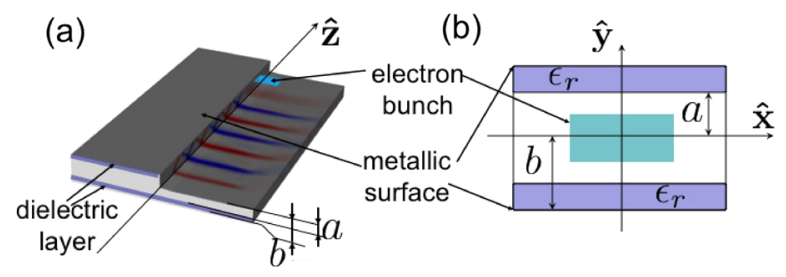

FIG. 1. Overview of the DLW structure geometry (left) and transverse cross section (right). A rectangular-shaped drive beam is displayed in blue.

$$
\lambda(x, y, z)=\sum_{m} \lambda_{m} \cos \left(k_{x, m} x\right) \delta\left(y-y_{0}\right) \delta\left(z-z_{0}\right),
$$

where $\lambda_{m}$ 's are constants, $z_{0}=v t$ and the index $m$ is defined such that $k_{x, m} \equiv(2 m+1) \frac{\pi}{L_{x}}$. In the charge-free vacuum region the electromagnetic wakefield satisfies the wave equation,

$$
\left(\nabla^{2}-\frac{1}{c^{2}} \frac{\partial^{2}}{\partial^{2} t}\right) \mathbf{E}=0
$$

In this paper we consider only the propagating modes with harmonic longitudinal and temporal dependencies of the form $\mathbf{E}(x, y, z, t)=\mathbf{E}(x, y) e^{i\left(\omega t-k_{z} z\right)} \quad$ [14,25], where $k_{z}=\mathbf{k} . \hat{\mathbf{z}}$. In addition to the propagating-mode solutions, Eq. (2) when subject to the boundary conditions also admits evanescent modes with imaginary eigenfrequencies $\omega$ [25]. Since these evanescent modes are present over short distances behind the drive bunch, they do not contribute to the beam dynamics of a subsequent witness bunch. These fields are consequently ignored in the remainder of this paper.

In the ultrarelativistic regime the synchronism condition is achieved because the wakefield phase velocity $v_{\varphi} \equiv \frac{\omega}{k_{z}}=$ $v \approx c$ is very close to the velocity of the witness beam. Since the transverse wave vector $k_{\perp} \equiv \sqrt{k^{2}-k_{z}^{2}} \simeq k / \gamma=0$, the wave equation (2) can be written only in terms of the partial derivatives with respect to the transverse coordinates:

$$
\nabla_{\perp}^{2} \mathbf{E}(x, y)=0 .
$$

The symmetry of the drive-beam charge distribution and the boundary conditions at $x= \pm L_{x} / 2$ determine (up to constants) the analytical expression of the fields. In the vacuum region the electric field components are simple combinations of trigonometric and hyperbolic functions:

$$
\begin{aligned}
E_{x} & \propto \sin \left(k_{x, m} x\right) \cosh \left(k_{y} y\right), \\
E_{y} & \propto \cos \left(k_{x, m} x\right) \sinh \left(k_{y} y\right), \\
E_{z} & \propto \cos \left(k_{x, m} x\right) \cosh \left(k_{y} y\right) .
\end{aligned}
$$

The axial field $E_{z}$ associated with this set of solutions is symmetric with respect to the horizontal axis [i.e. $\left.E_{z}(x,-y, z)=E_{z}(x, y, z)\right]$; we henceforth refer to this set as "monopole" modes. Similarly, a set of fields with an antisymmetric axial field $\left[E_{z}(x,-y, z)=-E_{z}(x, y, z)\right]$ is obtained by substitution of $\sinh \left(k_{y} y\right)$ with $\cosh \left(k_{y} y\right)$ and vice versa. This latter set is termed as "dipole" modes in the remaining of this paper.

Inside the dielectric, the transverse wave number cannot be neglected:

$$
k_{\perp, m}^{2} \equiv k_{x, m}^{2}+k_{y}^{2} \approx \frac{\omega^{2}}{c^{2}}\left(\epsilon_{r}-1\right)>0,
$$

where $\epsilon_{r}$ is the relative electric permittivity of the dielectric medium. The boundary conditions at $y= \pm b$ and at 
$x= \pm L_{x} / 2$ determine the trigonometric form of the field expressions. Inside the dielectric region, there is no distinction between monopole and dipole modes and the electric field components are given by

$$
\begin{aligned}
& E_{x} \propto \sin \left(k_{x, m} x\right) \cos \left[k_{y}(b-y)\right], \\
& E_{y} \propto \cos \left(k_{x, m} x\right) \sin \left[k_{y}(b-y)\right], \\
& E_{z} \propto \cos \left(k_{x, m} x\right) \cos \left[k_{y}(b-y)\right] .
\end{aligned}
$$

The corresponding expressions for the magnetic field in the vacuum and dielectric regions can be easily obtained following a similar prescription.

Inspection of the $z$ component of the fields indicates that the normal modes cannot be categorized in the usual transverse electric or transverse magnetic sets. The reason is that the separation surface between the dielectric and vacuum regions is in the $x-z$ plane unlike the case of the uniformly filled waveguides where this surface is in the transverse $x-y$ plane.

Therefore, it is natural to categorize the modes depending on the orientation of the fields with respect to the dielectric surface. Following the definition introduced in Ref. [28] we classify the mode as longitudinal section magnetic (LSM) and longitudinal section electric (LSE) modes corresponding, respectively, to the case when the magnetic and electric field component perpendicular to the dielectric surface vanishes. In the configuration shown in Fig. 1, LSE and LSM modes correspond, respectively, to $E_{y}=0$ and $H_{y}=0$ at the vacuum-dielectric interface.

\section{DISPERSION EQUATIONS AND ELECTROMAGNETIC WAKEFIELDS}

\section{A. Longitudinal section magnetic (LSM) modes}

To obtain the normal mode frequencies it is convenient to use the Hertzian potential vector method [28]. For example, to determine the LSM modes $\left(H_{y}=0\right.$ in vacuum region), it is convenient to exploit that $\boldsymbol{\nabla} \cdot \mathbf{B}=0$ and to express the fields in terms of the yet-undetermined Hertzian electric vector potential $\boldsymbol{\Pi}_{\mathbf{e}}$ as

$$
\mathbf{H}=i \omega \epsilon \boldsymbol{\nabla} \times \boldsymbol{\Pi}_{\mathbf{e}} .
$$

The curl equation for the electric field implies that

$$
\mathbf{E}=k^{2} \mathbf{\Pi}_{\mathbf{e}}+\nabla \Phi
$$

where $\nabla \Phi$ is an arbitrary scalar function. A relationship between the vector potential $\boldsymbol{\Pi}_{\mathbf{e}}$ and the scalar potential $\Phi$ can be obtained from the previous two equations and the Maxwell's equation for the curl of the magnetic field:

$$
\nabla\left(\nabla \cdot \boldsymbol{\Pi}_{\mathbf{e}}\right)-\nabla^{2} \boldsymbol{\Pi}_{e}=k^{2} \boldsymbol{\Pi}_{\mathbf{e}}+\nabla \Phi .
$$

At this point, in a similar way as for the standard potentials $(\boldsymbol{\phi}, \mathbf{A})$, a suitable gauge would decouple the equations for the vector and scalar Hertzian potentials. The Lorentz-type condition $\boldsymbol{\nabla} \cdot \boldsymbol{\Pi}_{\mathbf{e}}=\Phi$ makes the vector potential to obey the homogeneous Helmholtz equation,

$$
\nabla^{2} \boldsymbol{\Pi}_{\mathbf{e}}+k^{2} \boldsymbol{\Pi}_{e}=0 .
$$

A similar equation can be derived for the scalar potential $\Phi$ assuming free-space wave propagation $(\boldsymbol{\nabla} \cdot \mathbf{E}=0)$. From the previous equation and the Lorentz gauge condition, the electric field in Eq. (8) can be solely rewritten in terms of $\boldsymbol{\Pi}_{\mathbf{e}}$ as

$$
\mathbf{E}=k^{2} \boldsymbol{\Pi}_{\mathbf{e}}+\nabla\left(\nabla \cdot \boldsymbol{\Pi}_{\mathbf{e}}\right) .
$$

In this case the Hertzian vector potential is simply proportional with the standard vector potential: $\mathbf{A}=\frac{i \omega}{c^{2}} \boldsymbol{\Pi}_{\mathbf{e}}$.

The boundary conditions at the perfectly conducting walls $x= \pm \frac{L}{2}$ require $\hat{\mathbf{x}} \cdot \mathbf{H} \propto \hat{\mathbf{x}} \cdot\left(\boldsymbol{\nabla} \times \boldsymbol{\Pi}_{\mathbf{e}}\right)=0$. Since the $y$ component of the magnetic field must also vanish (LSM modes), only the $y$ component of the Hertzian vector potential is nonvanishing and

$$
\boldsymbol{\Pi}_{\mathbf{e}}=\psi_{e}(x, y) e^{i\left(\omega t-k_{z} z\right)} \hat{\mathbf{y}},
$$

where $\psi_{e}(x, y)$ is a function yet to be determined. From Eqs. (7) and (11) all fields can be expressed in terms of the unknown function $\psi_{e}(x, y)$ :

$$
\begin{aligned}
& E_{x}=\frac{\partial^{2} \psi_{e}}{\partial x \partial y}, \quad H_{x}=-\epsilon k_{z}^{2} c \psi_{e}, \quad E_{y}=k^{2} \psi_{e}+\frac{\partial^{2} \psi_{e}}{\partial y^{2}}, \\
& H_{y}=0, \quad E_{z}=-i k_{z} \frac{\partial \psi_{e}}{\partial y}, \quad H_{z}=i \epsilon k_{z} c \frac{\partial \psi_{e}}{\partial x} .
\end{aligned}
$$

The expression for $\psi_{e}$ that satisfies Eqs. (5) and (13), and the boundary conditions for the electric field at $x= \pm \frac{L}{2}$ is given by

$\psi_{e}= \begin{cases}A \cos \left(k_{x, m} x\right) \sinh \left(k_{x, m} y\right), & 0<y<a \\ B \cos \left(k_{x, m} x\right) \cos \left[k_{y}(b-y)\right], & a<y<b,\end{cases}$

where $A$ and $B$ are constants. Since $H_{x}\left(\propto \epsilon \psi_{e}\right)$ and $E_{z}\left(\propto \frac{\partial \psi_{e}}{\partial y}\right)$ are continuous at the dielectric-vacuum-region interface, the constants $A$ and $B$ can be eliminated thereby yielding the dispersion equation,

$$
\operatorname{coth}\left(k_{x, m} a\right) \cot \left[k_{y}(b-a)\right]=\frac{k_{y}}{\epsilon_{r} k_{x, m}} .
$$

For each discrete value of $k_{x, m}$ there is an infinite set of discrete $k_{y, n}$ values where $n$ is an integer. So, the eigenfrequencies are indexed by the integer couple $(m, n)$ and verify

$$
k_{x . m}^{2}+k_{y, n}^{2}=\frac{\omega_{m, n}^{2}}{c^{2}}\left(\epsilon_{r}-1\right) .
$$

It is worthwhile noting that the boundary conditions do not completely determine either the function $\psi_{e}$ or the fields. The reason being that, up to this point, the field source terms $(\rho$, j) were not taken into account although their symmetries were invoked. Still, it is straightforward to show that all fields (and also $\psi_{e}$ ) associated to a certain mode depend on a common normalization constant, the amplitude $E_{0 ; m, n}$, which remains to be determined. The expressions of the fields are given by 


$$
\begin{aligned}
& E_{x, m, n}= \begin{cases}-\frac{i E_{0 ; m, n} k_{x, m}}{k_{z}} \sin \left(k_{x, m} x\right) \cosh \left(k_{x, m} y\right) & 0<y<a \\
-\frac{i E_{0 ; m, n} k_{x, m}}{k_{z}} \frac{\cosh \left(k_{x, m} a\right)}{\sin \left[k_{y, n}(b-a)\right]} \sin \left(k_{x, m} x\right) \sin \left[k_{y, n}(b-y)\right] & a<y<b\end{cases} \\
& E_{y, m, n}= \begin{cases}\frac{i E_{0, m, n}, k^{2}}{k_{x, m} k_{z}}\left(k_{x, m}^{2}+k_{z}^{2}\right) \cos \left(k_{x, m} x\right) \sinh \left(k_{x, m} y\right) & 0<y<a \\
\frac{i E_{0 ; m, n} \cosh \left(k_{x, m} a\right)}{k_{y, n} k_{z} \sin \left[k_{y, n}(b-a)\right]}\left(k_{z}^{2}+k_{x, m}^{2}\right) \cos \left(k_{x, m} x\right) \cos \left[k_{y, n}(b-y)\right] & a<y<b\end{cases} \\
& E_{z, m, n}= \begin{cases}E_{0 ; m, n} \cos \left(k_{x, m} x\right) \cosh \left(k_{x, m} y\right) & 0<y<a \\
E_{0 ; m, n} \frac{\cosh \left(k_{x, m} a\right)}{\sin \left[k_{y, n}(b-a)\right]} \cos \left(k_{x, m} x\right) \sin \left[k_{y, n}(b-y)\right] & a<y<b\end{cases} \\
& H_{x, m, n}= \begin{cases}-\frac{i E_{0 ; m, n} k_{z} \epsilon}{k_{x, m}} \cos \left(k_{x, m} x\right) \sinh \left(k_{x, m} y\right) & 0<y<a \\
-\frac{i E_{0, m, n} k_{z} \epsilon c}{k_{x, m}} \frac{\cosh \left(k_{x, m} a\right)}{\sin \left[k_{y, n}(b-a)\right]} \cos \left(k_{x, m} x\right) \cos \left[k_{y, n}(b-y)\right] & a<y<b\end{cases} \\
& H_{y, m, n}= \\
& H_{z, m, n}= \begin{cases}E_{0 ; m, n} \epsilon c \sin \left(k_{x, m} x\right) \sinh \left(k_{x, m} y\right) & a<y<b . \\
\frac{E_{0 ; m, n} k_{x, m} \epsilon c}{k_{y, n}} \frac{\cosh \left(k_{x, m} a\right)}{\sin \left[k_{y, n}(b-a)\right]} \sin \left(k_{x, m} x\right) \cos \left[k_{y, n}(b-y)\right] & \end{cases}
\end{aligned}
$$

\section{B. Longitudinal section electric (LSE) modes}

The case of the LSE modes $\left(E_{y}=0\right)$ can be treated in the same way as the LSM modes. The similarity comes from the fact that $\boldsymbol{\nabla} \cdot \mathbf{E}=0$ in free space. The Hertzian vector electric potential is replaced by a vector magnetic potential $\boldsymbol{\Pi}_{h}$ which is related to the fields in a similar way as the vector electric potential in Eqs. (7) and (11):

$$
\mathbf{E}=-i \omega \mu \boldsymbol{\nabla} \times \boldsymbol{\Pi}_{\mathbf{h}}
$$

$$
\mathbf{H}=k^{2} \boldsymbol{\Pi}_{\mathbf{h}}+\nabla\left(\nabla \cdot \boldsymbol{\Pi}_{\mathbf{h}}\right) .
$$

As in the previous case, it is convenient to factor out the $t$ and $z$ dependencies of the $\boldsymbol{\Pi}_{\mathbf{h}}$ and to define a scalar function $\psi_{h}(x, y): \boldsymbol{\Pi}_{\mathbf{h}}=\psi_{h}(x, y) e^{i\left(\omega t-k_{z} z\right)} \hat{\mathbf{y}}$. It is straightforward to derive the dispersion equation for the LSE modes,

$$
\operatorname{coth}\left(k_{x} a\right) \cot \left[k_{y}(b-a)\right]=-\frac{k_{x}}{k_{y}},
$$

and the expressions for the electromagnetic-field components:

$$
\begin{aligned}
& E_{x, m, n}= \begin{cases}\frac{i E_{0, m, n} k_{z}}{k_{x, m}} \sin \left(k_{x, m} x\right) \cosh \left(k_{x, m} y\right) & 0<y<a \\
\frac{i E_{0 ; m, n} k_{z}}{k_{x, m}} \frac{\cosh \left(k_{x, m} a\right)}{\sin \left[k_{y, n}(b-a)\right]} \sin \left(k_{x, m} x\right) \sin \left[k_{y, n}(b-y)\right] & a<y<b\end{cases} \\
& E_{y, m, n}=0 \\
& E_{z, m, n}= \begin{cases}E_{0 ; m, n} \cos \left(k_{x, m} x\right) \cosh \left(k_{x, m} y\right) & 0<y<a \\
E_{0 ; m, n} \frac{\cosh \left(k_{x, m} a\right)}{\left.\sin \left[k_{y, n} b-a\right)\right]} \cos \left(k_{x, m} x\right) \sin \left[k_{y, n}(b-y)\right] & a<y<b\end{cases} \\
& H_{x, m, n}= \begin{cases}\frac{i E_{0 ; m, n} k_{x, m}}{k_{z} \mu c} \cos \left(k_{x, m} x\right) \sinh \left(k_{x, m} y\right) & 0<y<a \\
-\frac{i E_{0 ; m, n} k_{y, n}}{k_{z} \mu c} \frac{\cosh \left(k_{x, m} a\right)}{\sin \left[k_{y, n}(b-a)\right]} \cos \left(k_{x, m} x\right) \cos \left[k_{y, n}(b-y)\right] & a<y<b\end{cases} \\
& H_{y, m, n}= \begin{cases}\frac{i E_{0 ; m, n}\left(k_{x, m}^{2}+k_{z}^{2}\right)}{k_{x, m} k_{z} \mu c} \sin \left(k_{x, m} x\right) \cosh \left(k_{x, m} y\right) & 0<y<a \\
\frac{i E_{0: m, n}\left(k_{x, m}^{2}+k_{z}^{2}\right)}{k_{x, m} k_{z} \mu c} \frac{\cosh \left(k_{x, m} a\right)}{\sin \left[k_{y, n}(b-a)\right]} \sin \left(k_{x, m} x\right) \cos \left[k_{y, n}(b-y)\right] & a<y<b\end{cases} \\
& H_{z, m, n}= \begin{cases}\frac{E_{0: m, n}}{\mu c} \sin \left(k_{x, m} x\right) \sinh \left(k_{x, m} y\right) & a<y<b . \\
-\frac{E_{0 ; m, n} k_{y}}{k_{x, m} \mu c} \frac{\cosh \left(k_{x, m} a\right)}{\sin \left[k_{y, n}(b-a)\right]} \sin \left(k_{x, m} x\right) \cos \left[k_{y, n}(b-y)\right] & \end{cases}
\end{aligned}
$$

As in the LSM case, the gauge condition between the Hertzian magnetic vector potential $\left(\boldsymbol{\Pi}_{\mathbf{h}}\right)$ and its associated scalar potential $(\Phi)$ is also similar to the Lorentz gauge applied to standard potentials. For the LSE mode, the relationships between the Hertzian and the conventional $(\phi, \mathbf{A})$ potentials are 


$$
\phi=i \omega \Phi \quad \mathbf{A}=\mu \boldsymbol{\nabla} \times \boldsymbol{\Pi}_{\mathbf{h}}+\nabla\left(\nabla \cdot \boldsymbol{\Pi}_{\mathbf{h}}\right) .
$$

The motivation for splitting the modes into LSM and LSE relies on the orthogonality relation [28] between pairs of transverse field components

$$
\iint_{S} \mathbf{E}_{\perp, n} \times \mathbf{H}_{\perp, m} \cdot \hat{\mathbf{z}} d a=0 \quad n \neq m,
$$

with $\mathbf{E}_{\perp, n}$ and $\mathbf{B}_{\perp, n}$ being the transverse electric and magnetic fields corresponding to two arbitrary modes generically denoted by $n$ and $m$, respectively. The integration is extended over the transverse area of the dielectric structure. It is important to note that the set of eigenfunctions derived in this paper forms a complete set only when the evanescent modes with imaginary eigenfrequencies (not derived here) are also included [25].

\section{WAKEFIELD AMPLITUDES}

Although we have obtained the general expression for the electromagnetic field, the constants $E_{0 ; m, n}$ still remained to be determined. An often used method to find the constants $E_{0 ; m, n}$ in Eqs. (17) and (21), is to evaluate the Green function of the wave equation with sources included. The latter is usually a cumbersome procedure and we instead chose to determine $E_{0 ; m, n}$ 's based on energy balance considerations. The electromagnetic energy stored in the DLW equals the mechanical work performed on the drive bunch. For a linear wakefield, the decelerating field $E_{d}$ acting on a drive point-charge is half of the wakefield amplitude, i.e. $E_{d}=\frac{E_{0: m, n}}{2}$ as a consequence of the fundamental wakefield theorem [29]. Suppose the drive charge moves over an infinitely short distance $\delta z$. The work performed by the wakefield on the drive charge should equal the energy stored in the field, i.e.,

$$
\sum_{m, n} \int \delta\left(y-y_{0}\right) \frac{E_{z ; m, n}(z=v t)}{2} d x d y \delta z=\frac{1}{2} \int\left(\epsilon E^{2}+\mu_{0} H^{2}\right) d x d y \delta z
$$

where the integration extends over the transverse plane. It is straightforward to evaluate the full expressions of the wakefield amplitudes in terms of the wave numbers $k_{x, m}$ and $k_{y, n}$ from Eqs. (24), (17), and (21):

$$
\begin{aligned}
E_{0 ; m, n}^{\mathrm{LSM}} & =\frac{1}{2 \epsilon_{0}} \frac{\lambda_{m} \cosh \left(k_{x, m} y_{0}\right)}{\frac{\sinh \left(2 k_{x, m} a\right)}{2 k_{x, m}}+\frac{\epsilon_{r} \cosh ^{2}\left(k_{x, m} a\right)}{\sin ^{2}\left[k_{y, n}(b-a)\right]}\left\{\frac{b-a}{2}\left(1+\frac{\epsilon_{r} k_{x, m}^{2}}{k_{y, n}^{2}}\right)-\frac{\sin \left[2 k_{y, n}(b-a)\right]}{4 k_{y, n}}\left(1-\frac{\left.\epsilon_{r} k_{x, m}^{2}\right)}{k_{y, n}^{2}}\right)\right\}} \\
E_{0 ; m, n}^{\mathrm{LSE}} & =\frac{1}{2 \epsilon_{0}} \frac{\lambda_{m} \cosh \left(k_{x, m} y_{0}\right)}{\frac{\sinh \left(2 k_{x, m} a\right)}{2 k_{x, m}}+\frac{\cosh ^{2}\left(k_{x, m} a\right)}{\sin ^{2}\left[k_{y, n}(b-a)\right]}\left\{\frac{b-a}{2}\left(\epsilon_{r}+\frac{k_{y, n}^{2}}{k_{x, m}^{2}}\right)-\frac{\sin \left[2 k_{y, n}(b-a)\right]}{4 k_{y, n}}\left(\epsilon_{r}-\frac{k_{y, n}^{2}}{k_{x, m}}\right)\right\}} .
\end{aligned}
$$

The field amplitudes for the dipole modes can be obtained from the previous equations by substituting $\sinh \left(k_{x, m} u\right)$ for $\cosh \left(k_{x, m} u\right)$ where $u$ takes on the values of $a$ and $y_{0}$. As expected, the wakefield amplitude scales linearly with the drive-bunch charge and inverse proportionally with the transverse size of the structure. To this point this model is fully three dimensional and the only limitation stems from the assumed symmetry of the transverse drive charge distribution with respect to the $y$ axis.

\section{COMPARISON WITH FDTD SIMULATIONS}

\section{A. Three-dimensional case}

To evaluate the wakefield associated to a drive bunch, an integration over the full three-dimensional continuous charge distribution must be performed. In practice the integration is replaced by numerical summations of discrete charge distributions similar to those described by Eq. (1). This process is similar to the charge discretization procedure used in standard PIC algorithms [30]. An important feature of this model is that the integration over $x$ direction is already performed through the Fourier expansion of the charge distribution. For the charge distributions considered in this paper, only a few Fourier terms $(<10)$ are sufficient to obtain an accurate representation of the distribution along the $x$ direction. This is significantly less than the number of grid points in $x$ direction needed by most PIC codes to evaluate the 3D-collective effects and external fields.

The integration over the vertical direction is straightforward and in most cases, when the drive charge distribution is also symmetric with respect to the horizontal axis, the contribution of the dipole modes cancels out.

Since the phase velocity of the wakefield is the same as the velocity of the drive beam, causality principle requires that the wakefield vanishes ahead of the drive charge $[29,31,32]$. Therefore, the integration over the longitudinal direction extends only from the observation point to the actual drive charge position.

In general, the charge density of the drive beam can be written as $\rho(x, y, z)=\int \lambda\left(x, y, z ; y_{0}, z_{0}\right) f\left(y_{0}, z_{0}\right) d y_{0} d z_{0}$, where $f(y, z)$ is the charge density in the $y-z$ subspace and $\lambda(x, y, z)$ is the same as in Eq. (1). The longitudinal wakefield assumes the form

$$
W_{z}(z)=\sum_{m=0,1, \cdots} \sum_{n=0,1, \cdots} \int_{z}^{\infty} \rho\left(z^{\prime}\right) W_{z, m, n}\left(z-z^{\prime}\right) d z^{\prime}
$$


where $W_{z}$ stands for the longitudinal electric field at the witness beam position, $\rho\left(z^{\prime}\right)=\int d x d y \rho\left(x, y, z^{\prime}\right)$ is the longitudinal charge distribution, and $W_{z, m, n}$ is the longitudinal electric field component associated to either LSM or LSE modes [Eqs. (17) and (21)]. The summation is performed over all excited modes.

The witness beam dynamics is determined by the Lorentz force generated by a typically much larger drive-beam charge which moves ahead of it with a constant ultrarelativistic speed. It is straightforward to show based on Maxwell equations that the curl of the Lorentz force acting on a unit charge pointlike witness beam can be related to the total derivative of the magnetic field [33]:

$$
\boldsymbol{\nabla} \times(\mathbf{E}+\mathbf{v} \times \mathbf{B})=-\frac{d \mathbf{B}}{d t} .
$$

In most practical applications the witness beam is also ultrarelativistic and it is moving at a constant distance behind the drive beam. Therefore the fields "seen" by the witness charge are constant $\left(\frac{d \mathbf{B}}{d t}=0\right)$ and so, based on the previous equation, the witness charge motion takes place in a quasiconservative external field. Wakefields are defined as the Lorentz force acting on a unit charge witness beam $\mathbf{W} \equiv \mathbf{E}+\mathbf{v} \times \mathbf{B}$. For the "stationary" case described above, $\boldsymbol{\nabla} \times \mathbf{W}=0$ and the curl operator can be split into its transverse and longitudinal components to obtain the result from PanofskyWenzel theorem:

$$
\boldsymbol{\nabla}_{\perp} W_{z}=-\frac{\partial \mathbf{W}_{\perp}}{\partial \zeta}
$$

where $\zeta \equiv z-v t$. Although the transverse wakefields can be directly obtained from Eqs. (17) and (21), they can also be obtained more easily from Panofsky-Wenzel theorem which also offers some valuable physical insights. An interesting example is when the drive-beam charge consists of an infinitely long linear distribution. In this limit $k_{x} \rightarrow 0$ and it is easy to show that the longitudinal wakefield does not depend on the transverse coordinates. Consequently, due to Panofsky-Wenzel theorem, the transverse wakefields vanish. This case will be extensively examined in Sec. VC in connection with the possible use of flatbeams.

The results obtained from Eq. (26) are benchmarked against simulations performed with VORPAL a conformal FDTD PIC electromagnetic solver [34]. VORPAL is a parallel, object-oriented framework for three-dimensional relativistic electrostatic and electromagnetic plasma simulation. The DLW model implemented in VORPAL is fully three dimensional; see Fig. 2. The model consists of the rectangular DLW surrounded by perfectly conducting boundaries (PCBs), The lower and upper $z$ planes are terminated by PMLs that significantly suppresses artificial

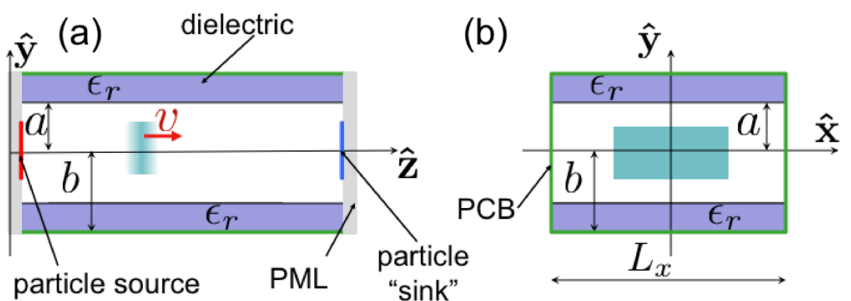

FIG. 2. Longitudinal (a) and transverse sections (b) of the DLW model implemented in VORPAL. The rectangular box delimited by green blocks represents the 3D computational domain used in the simulations. Grey, green, and purple blocks, respectively, stand for perfectly matched layer (PML), perfectly conducting boundary (PCB), and dielectric material (with associated relative dielectric permittivity $\epsilon_{r}$ ). The cyan rectangles represent the electron bunch distribution.

reflections of incident radiation [35]. A particle source located on the surface of the lower $z$ plane produced macroparticles uniformly distributed in the transverse plane $(x, y)$ and following a Gaussian longitudinal distribution with total charge $Q$ described by

$$
\begin{aligned}
\Xi(x, y, \zeta)= & \frac{Q}{\sqrt{2 \pi} \sigma_{z} w_{x} w_{y}} e^{-\left(\zeta^{2} / 2 \sigma_{z}^{2}\right)} H\left(\frac{w_{x}}{2}-|x|\right) \\
& \times H\left(\frac{w_{y}}{2}-|y|\right) H\left(3 \sigma_{z}-|\zeta|\right),
\end{aligned}
$$

where $w_{x}\left(w_{y}\right)$ is the full width transverse horizontal (vertical) beam size, and $\sigma_{z}$ the longitudinal root-meansquare (rms) length. The function $H(\cdots)$ is the Heaviside function. The longitudinal Gaussian distribution is truncated at $\pm 3 \sigma_{z}$. Finally, a "particle sink" at the upper $z$ plane allows macroparticles to exit the computational domain without being scattered or creating other source of radiation.

In order to precisely benchmark our model, a DLW which supports both LSM and LSE modes is chosen. The parameters of the structure and driving bunch are gathered in Table I and the frequency and the amplitude associated

TABLE I. Parameters of the DLW structure and drive bunch used for benchmarking our theoretical model with VORPAL simulations.

\begin{tabular}{lccc}
\hline \hline Parameter & Symbol & Value & Unit \\
\hline Vacuum gap & $a$ & 2.5 & $\mathrm{~mm}$ \\
Height & $b$ & 5.0 & $\mathrm{~mm}$ \\
Width & $L_{x}$ & 10.0 & $\mathrm{~mm}$ \\
Relative permittivity & $\epsilon_{r}$ & 4.0 & $\ldots$ \\
Bunch energy & $\mathcal{E}$ & 1 & $\mathrm{GeV}$ \\
Bunch charge & $Q$ & 1.0 & $\mathrm{nC}$ \\
rms bunch length & $\sigma_{z}$ & 1.0 & $\mathrm{~mm}$ \\
Bunch full width & $w_{x}$ & 6.0 & $\mathrm{~mm}$ \\
Bunch full height & $w_{y}$ & 4.0 & $\mathrm{~mm}$ \\
\hline \hline
\end{tabular}




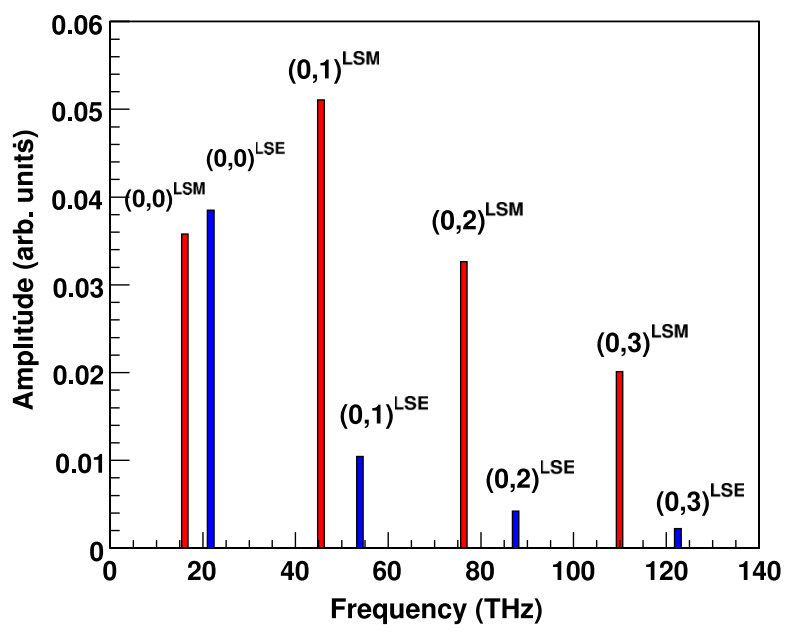

FIG. 3. Eigenfrequencies $\left[f_{m, n} \equiv \omega_{m, n} /(2 \pi)\right]$ and associated amplitudes for the mode induced in the DLW with parameter listed in Table I. The $(m, n)$ LSM and LSE modes are, respectively, shown as red and blue bars.

to the first few modes appear in Fig. 3. The drive-bunch energy is set to $\mathcal{E}=1.0 \mathrm{GeV}$ consistent with the ultrarelativistic approximation used in the analytical model.

The longitudinal component of the electric field simulated with VORPAL is shown in Fig. 4 as a two-dimensional projection in the $y-z$ plane.

The comparison between theoretical calculation and VORPAL simulations is shown in Figs. 5 and 6. The fields in Fig. 5 are evaluated as a function of the axial coordinate $z$ at a given transverse location $(x=3.0 \mathrm{~mm}, y=2.0 \mathrm{~mm}$ ) which corresponds to the upper-left corner of the charge distribution. Figure 6 displays the electromagnetic field evaluated as a function of the horizontal [Fig. 6 (left)] and

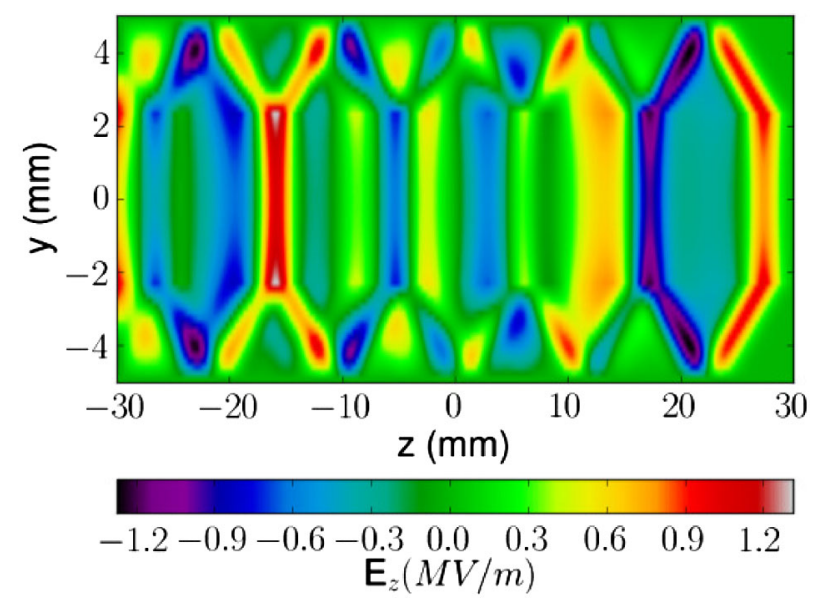

FIG. 4. Snapshot of the axial electric field $E_{z}(x=0, y, z)$ in the midplane of a slab DLW for the bunch and structure parameters shown in Table I. The center of the drive bunch is located at $z=$ $27 \mathrm{~mm}$ and it is moving in the positive $z$ direction. The field was obtained from VORPAL simulations.
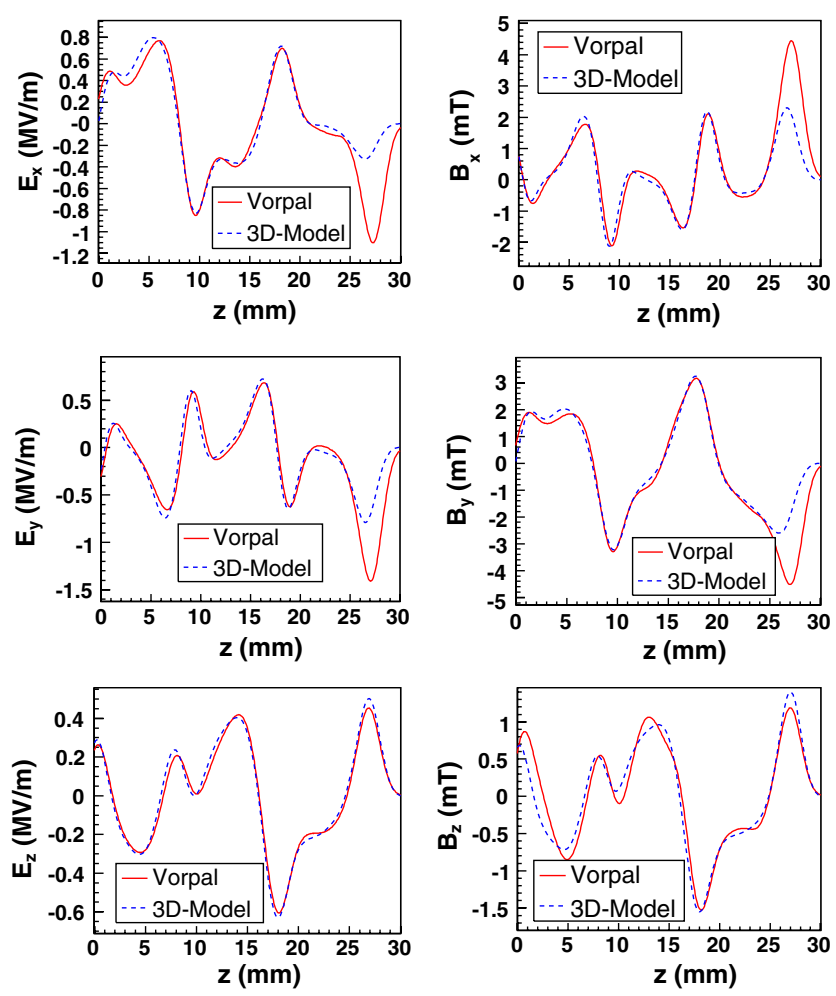

FIG. 5. Comparison of the electromagnetic-field components calculated with our model (blue dashed line) and simulated with VORPAL (red solid lines). The fields are computed on a line parallel to the $z$ axis with transverse offset $x=3.0 \mathrm{~mm}$ and $y=$ $2.0 \mathrm{~mm}$. The center of the drive beam is at $z=27.5 \mathrm{~mm}$. The DLW and bunch parameters are the ones displayed in Table I.

vertical [Fig. 6 (right)] transverse coordinate at a given axial location $z=18.6 \mathrm{~mm}$ corresponding to the minimum $E_{z}$ shown in Fig. 5 (i.e. maximum accelerating field). All plots show a decent agreement (relative discrepancy $<25 \%$ ) between our model and VORPAL simulations. The notable disagreement observed in Fig. 5 for the transverse fields in the vicinity of the driving charge (i.e. $z \simeq 27.5 \mathrm{~mm}$ ) is rooted in the absence of velocity fields in our model (only radiation field contributes to the wakefield) while the VORPAL simulations include both velocity and radiation fields. In fact given the bunch charge and duration, the amplitude of the velocity field can be evaluated by convolving the charge distribution with the electric field generated by an ultrarelativistic particle $\mathbf{E}(r, \zeta)=q /\left(2 \pi \epsilon_{0}\right) \times$ $\left(\mathbf{r}_{\perp} / r_{\perp}^{2}\right) \delta(\zeta)$, where $e$ and $\epsilon_{0}$ are, respectively, the electronic charge and vacuum permittivity, and $\mathbf{r}_{\perp} \equiv(x, y)$ and $\zeta$ are, respectively, the transverse and longitudinal coordinates of the observation point referenced to the particle's location. Such a convolution with Eq. (29) yields the transverse electric field components at $(x, y, \zeta)=\left(w_{x}, w_{y}, 0\right)$ to be $\sim 1 \mathrm{MV} / \mathrm{m}$ for the bunch parameter listed in Table I. The latter value is in agreement with the observed difference between the VORPAL and theoretical models; see $E_{x}$ and $E_{y}$ components in Fig. 6. 

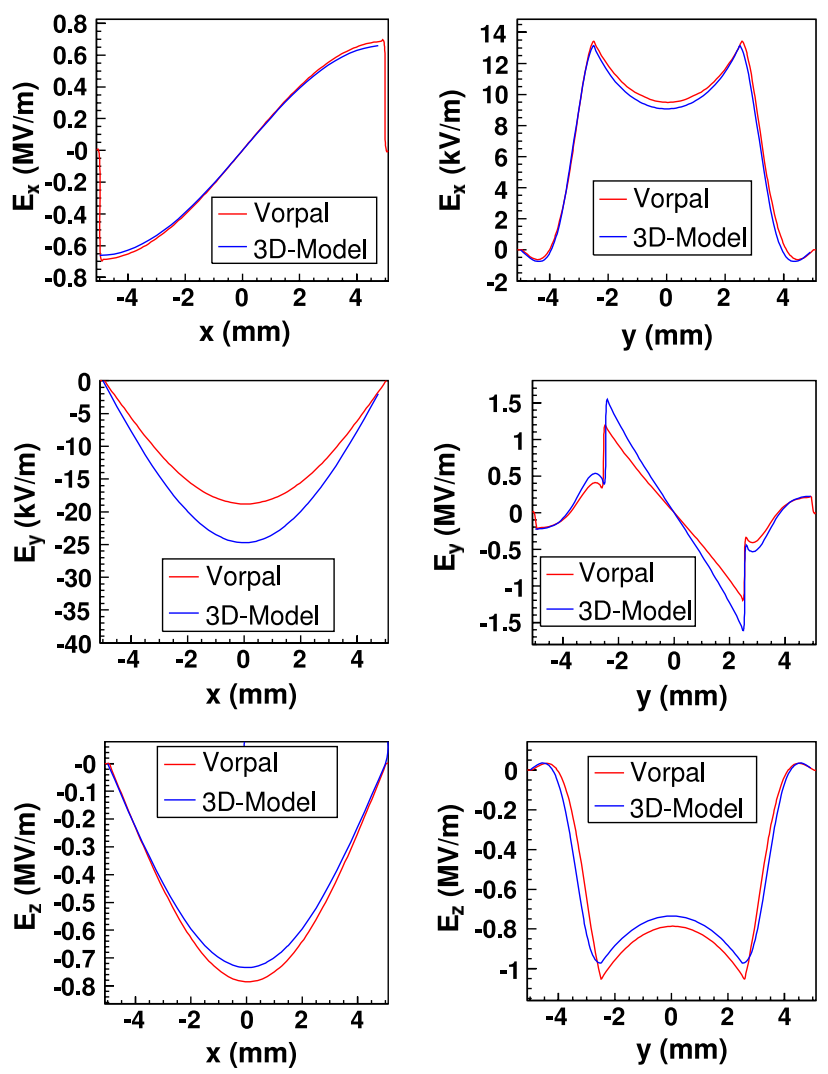

FIG. 6. Comparison of the electromagnetic-field components calculated with our model (blue dashed line) and simulated with VORPAL (red solid lines). The fields are computed at a given longitudinal position $z=18.6 \mathrm{~mm}$. The left-column plots display the dependence on the horizontal coordinate $x$ at $y=0.043 \mathrm{~mm}$ while the right-column plots show the dependence on the vertical coordinate $y$ at $x=0.043 \mathrm{~mm}$. The DLW and bunch parameters are the ones displayed in Table I.

\section{B. Witness-bunch dynamics}

In the previous section our primary focus has been on the wakefields generated within and downstream of the drive bunch. In this section we briefly address the wakefield produced by the witness bunch and especially the fields within the witness bunch. We refer to these fields as short-range wakefields. The analytical derivation of these wakefields is identical to the one detailed in the previous sections. Considering the case of the longitudinal wakefield, the total field produced by the drive and witness bunches can be computed in a straightforward manner by writing $\rho(z)$ in Eq. (26) as a sum of the drive bunch and witness bunch longitudinal charge distribution. In this section we take

$\rho(z)=\frac{Q}{\sqrt{2 \pi}}\left[\frac{1}{\sigma_{z, d}} e^{-\left[\left(z-z_{d}\right)^{2} / 2 \sigma_{z, d}^{2}\right]}+\frac{R}{\sigma_{z, w}} e^{-\left[\left(z-z_{w}\right)^{2} / 2 \sigma_{z, w}^{2}\right]}\right]$, where the first and second terms on the right-hand side refer, respectively, to the drive- and witness-bunch distributions. The parameters $\sigma_{z,[d, w]}$ and $z_{[d, w]}$ are the rms bunch lengths and positions, $Q$ is the drive-bunch charge, and $R Q$ is the witness-bunch charge so that $R$ is the witness-to-drive-bunch charge ratio. The value of $z_{d}$ is arbitrary but $z_{w}$ is chosen to ensure the witness bunch is located at an accelerating phase of the wakefield generated by the drive bunch.

Depending on the witness-bunch parameters, the shortrange wakefield can significantly alter the field seen by the particles within the witness bunch compared to that assumed in Sec. VA. Besides being dictated by the frontend application, the witness-bunch parameters are also constrained by the DLW accelerating mechanism. The witness-bunch length $\sigma_{z, w}$ should be much smaller than the typical wavelength of the accelerating field. Since the bunch duration (length) and charge of the witness bunch set the amplitude of the short-range wakefields, minimizing these fields implies that the charge should be limited. Going back to the academic case considered in the previous subsection, we compare in Fig. 7 the axial electric field seen by the witness-bunch particles for the same drive-bunch parameter as shown in Fig. 5. Two cases of witness-bunch length are considered $\left(\sigma_{z, w}=0.1,1 \mathrm{~mm}\right)$ and the charge ratio is varied $R \in$ $[0.01,0.1,0.2]$. For $R=0.01$, the field within the witness bunch is very close to the field obtained in the absence of witness bunch. As $R$ increases the short-range wake
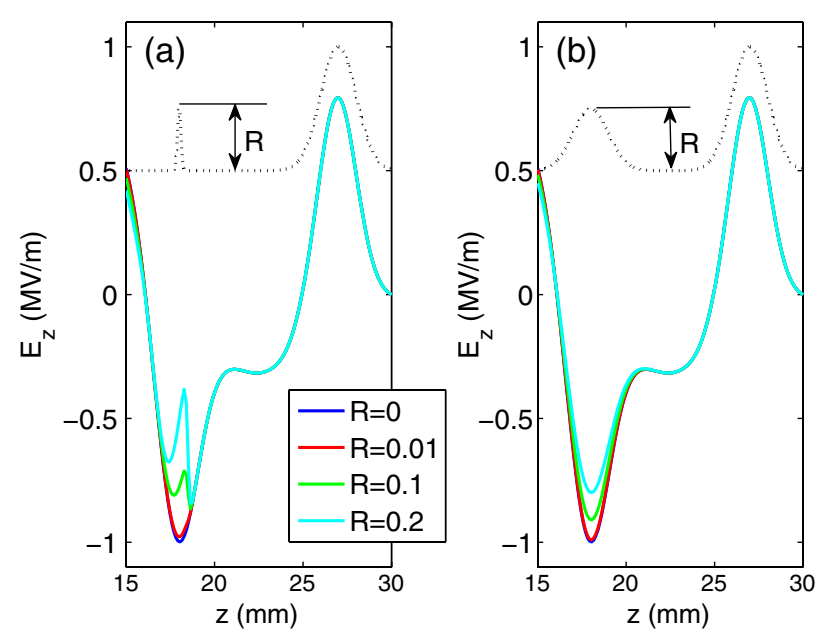

FIG. 7. Axial electric field generated by a charge distribution composed of a 1-nC drive bunch followed by a witness bunches with charge set to $R$ nC. Plots (a) and (b), respectively, correspond to a witness bunch with rms length of 0.1 and $1 \mathrm{~mm}$. The other parameters for the drive bunch are the ones displayed in Table I. The dotted trace represents the peak-normalized charge distribution considered for the field calculations (for sake of clarity the witness bunch is display for $R=0.5$ ). 
become important. As expected the effect is more pronounced for the shorter witness bunch. For the 100- $\mu \mathrm{m}$ witness bunch with $R=0.2$, the decelerating field inside the witness bunch is found to be comparable with the accelerating field produced by the drive bunch. Such an observation indicates that the witness-bunch dynamics is not significantly impacted by the short-range wakefield as long as $R \sim 0.01$.

The short-range wakefields are de facto included in the VORPAL simulations presented throughout this paper and in the numerical implementation of our model in Sec. VI.

\section{Two-dimensional limit}

An interesting limiting case occurs when $L_{x} \gg L_{y}$. In this "two-dimensional limit," the dependence of the fields on the horizontal coordinate $x$ is weak and completely vanishes when $L_{x} \rightarrow \infty$ (so that $k_{x} \simeq 0$ and $m=0$ ). In such a case the field amplitudes associated to the LSM and LSE modes are, respectively,

$$
E_{0 ; 0, n}^{\mathrm{LSM}} \simeq \frac{4 \Lambda}{a+\frac{\epsilon_{r}(b-a)}{\sin ^{2}\left[k_{y, n}(b-a)\right]}}, \quad \text { and } \quad E_{0 ; 0, n}^{\mathrm{LSE}} \simeq 0
$$

where $\Lambda$ is charge per unit length in the horizontal direction. The LSE modes are suppressed and the latter equation is in agreement with the results of Ref. [14].

This limit case can be practically reached by using structures with large aspect ratios $\left(L_{x} \gg L_{y}\right)$ driven by flat electron beams with their transverse horizontal emittance much smaller than the vertical one $\varepsilon_{x} \ll \varepsilon_{y}$. Flat beams can be produced in photoinjectors by using a roundto-flat beam transformation [36,37]. In such a scheme, a beam with large angular momentum is produced in a photoinjector [38]. Upon removal of the angular momentum by applying a torque with a set of skew quadrupole, the beam has its transverse emittance repartitioned with a tunable transverse emittances ratio [39]. Flat beams with

TABLE II. Parameter of the DLW structure and drive bunch used for benchmarking our theoretical model with VORPAL simulations.

\begin{tabular}{lccc}
\hline \hline Parameter & Symbol & Value & Unit \\
\hline Vacuum gap & $a$ & 100 & $\mu \mathrm{m}$ \\
Height & $b$ & 300 & $\mu \mathrm{m}$ \\
Width & $L_{x}$ & 10.0 & $\mathrm{~mm}$ \\
Relative permittivity & $\epsilon_{r}$ & 4.0 & $\ldots$ \\
Bunch energy & $\mathcal{E}$ & 1 & $\mathrm{GeV}$ \\
Bunch charge & $Q$ & 3.0 & $\mathrm{nC}$ \\
rms bunch length & $\sigma_{z}$ & 50 & $\mu \mathrm{m}$ \\
Full (rms) bunch width & $w_{x}\left(\sigma_{x}\right)$ & $3(0.870)$ & $\mathrm{mm}$ \\
Full (rms) bunch height & $w_{y}\left(\sigma_{y}\right)$ & $150(43.3)$ & $\mu \mathrm{m}$ \\
\hline \hline
\end{tabular}

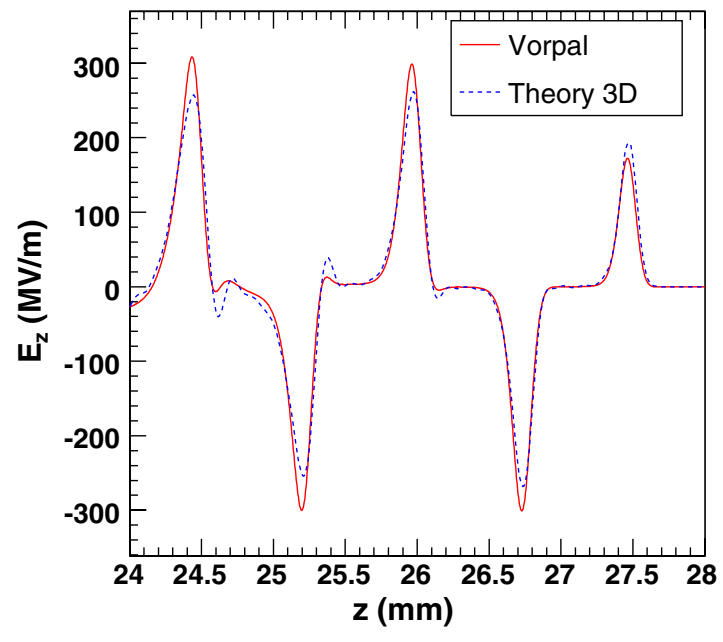

FIG. 8. Longitudinal electric field for a DLW with $L_{x} \gg L_{y}$ driven by a $3-\mathrm{nC}$ bunch. The bunch has a rectangular transverse shape with full width $w_{x}=3.0 \mathrm{~mm}$ and $w_{y}=150 \mu \mathrm{m}$, and a Gaussian longitudinal distribution with rms length $\sigma_{z}=50 \mu \mathrm{m}$. The theoretical model based on Eq. (31) (dashed blue line) is compared with VORPAL simulations (red line).

transverse emittance ratio of $\varepsilon_{x} / \varepsilon_{y} \simeq 100$ and aspect ratio of $\sim 20$ have been produced, even at a relatively low energy of $15 \mathrm{MeV}$ [27].

Based on the previous experience in producing flat beams and preliminary simulation of the Advanced Superconducting Test Accelerator (ASTA) currently in construction at Fermilab [40]. It is reasonable to consider a 3-nC flat beam generated from the ASTA photoinjector to have parameters tabulated in Table II when accelerated to $1 \mathrm{GeV}$. Considering a structure with $a=100 \mu \mathrm{m}$, $b=300 \mu \mathrm{m}$, and $\epsilon=4.0$ would yield a maximum axial wakefield amplitude of $\sim 300 \mathrm{MV} / \mathrm{m}$; see Fig. 8. The fundamental LSM frequency is $f_{0,0}=193 \mathrm{GH} z$ and the nearest LSE mode amplitude is $\sim 136$ times lower. In Fig. 8 the wakefield is computed with the asymptotic limit provided in Eq. (31) and is in excellent agreement with the FDTD simulations.

\section{IMPLEMENTATION IN A PARTICLE-IN-CELL BEAM DYNAMICS PROGRAM}

Although FDTD simulations provide important insight that can aid the design and optimization of the DLW geometry, their use to optimize a whole linear accelerator would be time and CPU prohibitive. Therefore it is worthwhile to include a semianalytical version of the model developed in this paper in a well-established beam dynamics program IMPACT-T [41]. Our main motivation toward this choice stems from the availability of a wide range of beam line elements models. In addition, IMPACT-T takes into account space-charge forces using a three-dimensional electrostatic solver. The algorithm consists in solving 
Poisson's equation in the bunch's rest frame and Lorentz boosting the computed electrostatic fields in the laboratory frame.

In a typical particle-tracking PIC program, like IMPACT-T, an electron bunch is described by a set of "macroparticles" arranged to mimic the bunch phase space distribution. Each macroparticle represents a large number of electrons (typically $10^{3}$ in our simulations). To evaluate the electrostatic fields in the bunch's rest frame, the macroparticles are deposited on the cells of a threedimensional grid. As a result of this charge deposition algorithm, the initial charge distribution is approximated by a set of point charges, located at the nodes of the threedimensional grid.

The line charge density corresponding to certain $y$ and $z$ coordinates is $\rho(x)=\sum_{x_{0}} q\left(x_{0}\right) \delta\left(x-x_{0}\right)$ and provided this distribution is symmetric the Fourier coefficients $\lambda_{m}$ from Eq. (1) are given by

$$
\lambda_{m}=\sum_{x_{0}} \frac{2 q\left(x_{0}\right) \cos \left(k_{x, m} x_{0}\right)}{L_{x}}
$$

In practice the linear charge distribution may not be "exactly" symmetric. In this case the charge at an arbitrary grid point $x_{0}$ is set at the average of the values given by the charge deposition algorithm at positions $x_{0}$ and $-x_{0}$.

The expressions of the electric and magnetic fields at a given position have the following form:

$$
F_{i}=\sum_{n} f_{i}(x, y ; n)\left(\sum_{z_{0}>z} e^{i k_{z}\left(z_{0}-z\right)} T_{i}\left(z_{0} ; n\right)\right)
$$

where $F$ can be either electric or magnetic field, $i$ is an index which indicates the field component, $n$ is the mode index, $f_{i}$ 's are the expressions of the fields from Eqs. (17) and (21) divided by the amplitude $E_{0, n}$, and

$$
T_{i}\left(z_{0} ; n\right)=\sum_{x_{0}, y_{0}} E_{0, n}\left(x_{0}, y_{0}, z_{0}\right),
$$

where $E_{0, n}$ 's are given by Eq. (25) with $\lambda_{m}$ 's given by Eq. (32) in which the charge is evaluated at the $\left(x_{0}, y_{0}, z_{0}\right)$ position.

The simulated electromagnetic-field components are compared with VORPAL simulations in Fig. 9 for the same case as presented in Fig. 5. In Fig. 9 the IMPACT-T simulations are performed with and without activating the space-charge algorithm. When accounting for spacecharge forces, IMPACT-T is in very good agreement with VORPAL.

The convolution over the $z$ summation in Eq. (33) can be performed with a numerical fast Fourier transformation.
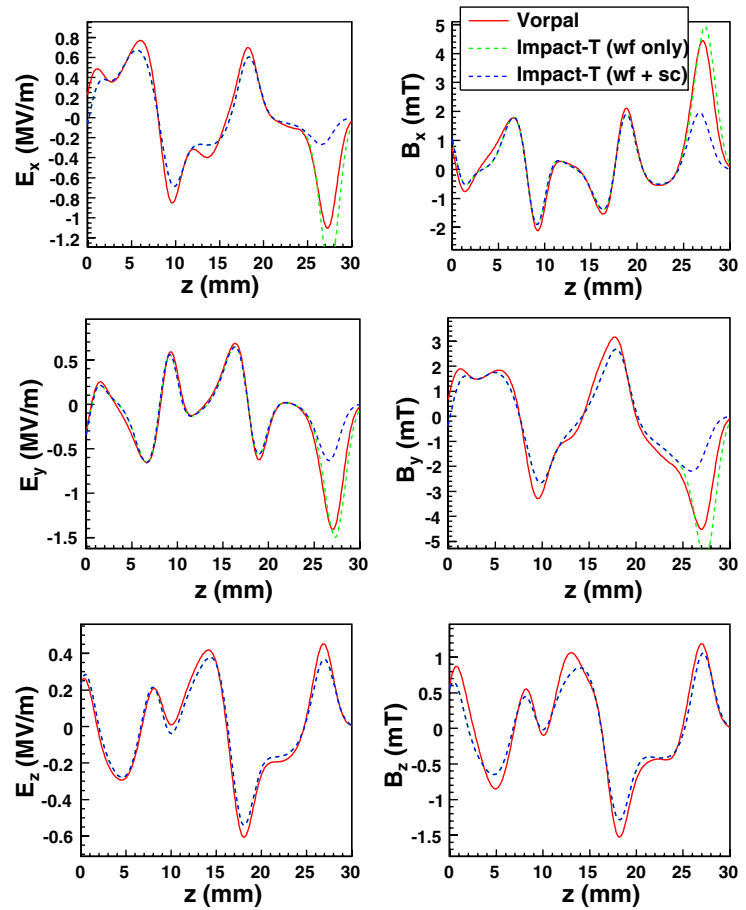

FIG. 9. Comparison of the electromagnetic-field components obtained from IMPACT-T [with (green dashed line) and without (blue dashed line) accounting for space-charge effects] with the fields simulated with VORPAL (red solid lines). The fields are computed on a line parallel to the $z$ axis with transverse offset $x=3.0 \mathrm{~mm}$ and $y=2.0 \mathrm{~mm}$. The center of the drive beam is at $z=27.5 \mathrm{~mm}$. The DLW and bunch parameters are the ones displayed in Table I.

Therefore the total number of operations needed to evaluate the wakefields is $\propto N_{\text {modes }} N_{x}^{2} N_{y}^{2} N_{z} \log N_{z}$. The total number of modes is twice the product between the modes allowed for each of the transverse wave numbers: $N_{\text {modes }}=$ $2 N_{k_{x}} N_{k_{y}}$. The factor of 2 comes from the inclusion of the dipole modes along with the vertically symmetric monopole modes. For the data generated in Fig. 9, the IMPACT-T simulations are more than 2 orders of magnitude faster than the VORPAL ones.

The altered version of IMPACT-T was used to explore a possible DLW experiment at the ASTA facility as a $1-\mathrm{GeV}$ electron beam is injected in a DLW. The beam and structure have the parameters displayed in Table II. For these simulations, the electron beam is an idealized cold beam without energy spread or divergence. Figure 10 compares the longitudinal phase spaces simulated with VORPAL (top row) and IMPACT-T (bottom row) at two axial locations. The agreement on the phase space structure developing as this nonoptimized beam propagates in the DLW is excellent. The distortion of the longitudinal phase space associated to the witness bunch stems from the chosen long pulse duration (length of $0.3 \mathrm{~mm}$ ) compared with the wavelength of the fundamental mode $(\lambda=1.6 \mathrm{~mm})$. The mean and $\mathrm{rms}$ energies 

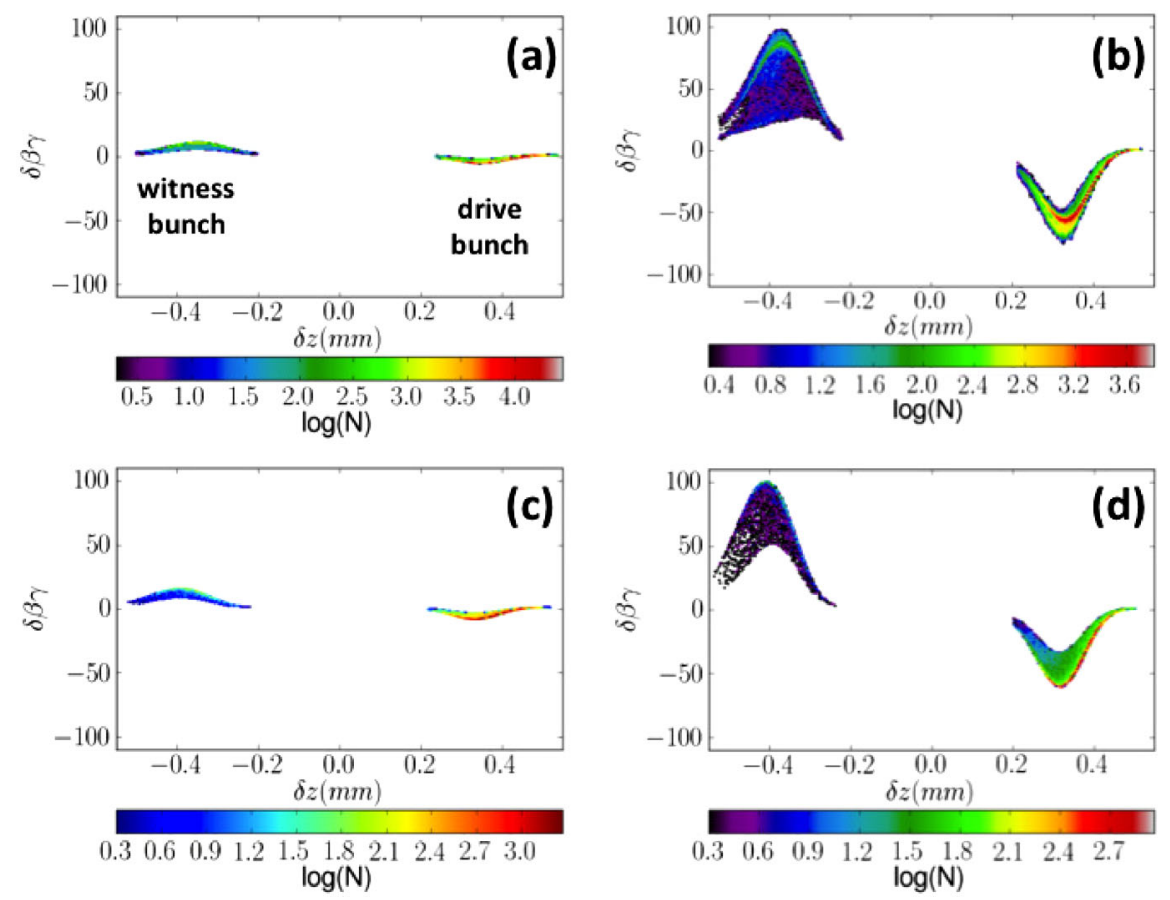

FIG. 10. Longitudinal phase spaces $[\delta z \equiv z-\langle z\rangle, \delta \beta \gamma \equiv \beta \gamma-\mathcal{E} /(m c)]$ snapshots as a 3-nC drive bunch followed by a 30-pC witness bunch of 1-ps duration, propagate in a DLW structure at $\langle z\rangle \simeq 2.3 \mathrm{~cm} \mathrm{(a),} \mathrm{(c)} \mathrm{and}\langle z\rangle \simeq 22 \mathrm{~cm}$ (b), (d) from the structure's entrance. Density plots (a), (b) and (c), (d), respectively, correspond to simulations carried with VORPAL and IMPACT-T. The beam energy is $\mathcal{E}=1 \mathrm{GeV}$. The DLW and drive-bunch parameters are the ones appearing in Table II. The logarithms in the plots are in decimal base.

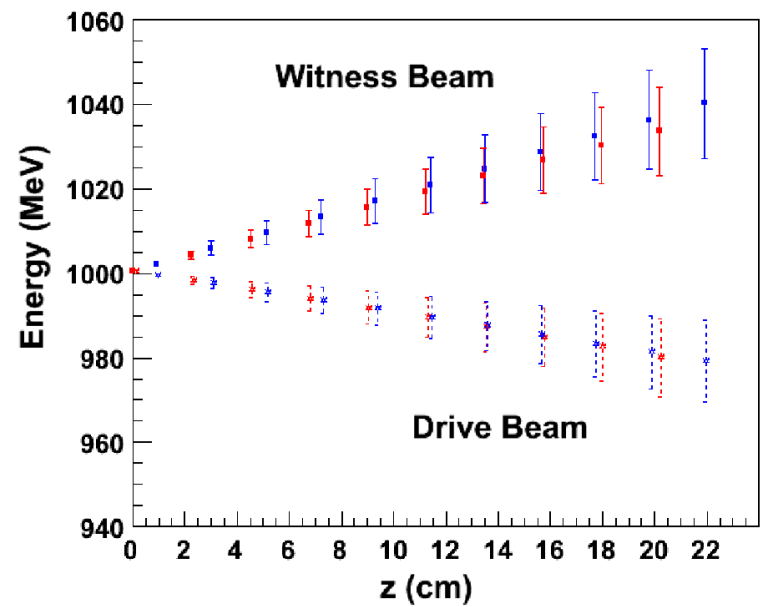

FIG. 11. Evolution of mean (symbols) and rms (bars) energy as the drive (top traces) and witness (bottom traces) bunches propagate along the DLW structure. The simulations are carried with VORPAL (red traces and symbols) and the modified version of IMPACT-T (blue traces and symbols). The beam energy is $\mathcal{E}=1 \mathrm{GeV}$. The DLW and bunch parameters are the ones gathered in Table II. The ordinate $z=0 \mathrm{~cm}$ corresponds to the DLW entrance.

evolution for the witness and drive bunches reported in Fig. 11 confirms the quantitative agreement between the FDTD and semianalytical models. Both models give mean and rms energies in agreement within $\lesssim 5 \%$.

\section{CONCLUSIONS}

In this paper we presented a three-dimensional model to evaluate wakefields in slab-symmetric dielectric-lined waveguides. The only limitation of this model stems from the assumed charge distribution symmetry with respect to the vertical axis $[\rho(x)=\rho(-x)]$. The model was successfully validated against three-dimensional FDTD PIC simulations performed with VORPAL and was implemented in the popular beam dynamics tracking program IMPACT-T. The added capability to IMPACT-T enables startto-end simulation of linear accelerators based on DLW accelerating structures. Furthermore, because IMPACT-T includes a space-charge algorithm, the upgraded version provides a valuable tool for investigating the performances of DLW acceleration when the dynamics of either, or both, of the drive and witness bunches is significantly impacted by space-charge effects. The observed good agreement between the developed algorithm and simulations performed with the VORPAL FDTD PIC program demonstrates that our model strikes an appropriate balance between efficiency, accuracy, and simplicity.

\section{ACKNOWLEDGMENTS}

We are thankful to J. Qiang and R. Rynes of LBNL for providing us with the sources of the IMPACT-T program. This work was supported by the Defense Threat Reduction 
Agency, Basic Research Award No. HDTRA1-10-1-0051, to Northern Illinois University. The work of D. M. is partially supported by the Fermilab Research Fellowship program under the Department of Energy Contract No. DE-AC02-07CH11359 with the Fermi Research Alliance, LLC.

[1] W. Wuensch et al., in Proceedings of the 2003 Particle Accelerator Conference (PAC03), Portland, Oregon (IEEE, New York, 2003), p. 495.

[2] B. Aune et al., Phys. Rev. ST Accel. Beams 3, 092001 (2000).

[3] W. Leemans, B. Nagler, A. J. Gonsalves, Cs. Tóth, K. Nakamura, C. G. R. Geddes, E. Esarey, C. B. Schroeder, and S. M. Hooker, Nature Phys. 2, 696 (2006).

[4] A. C. Melissinos, arXiv:physics/0410273.

[5] G. A. Voss and T. Weiland, DESY Report No. M-82-10, 1982; W. Bialowons et al., in Proceedings of the 1988 European Particle Accelerator Conference (EPAC 1988), Rome, Italy (1988), p. 902.

[6] I. Blumenfeld et al., Nature (London) 445, 741 (2007).

[7] K-. Y. Ng, Phys. Rev. D 42, 1819 (1990).

[8] J. B. Rosenzweig, A. M. Cook, A. Scott, M. C. Thompson, and R. B. Yoder, Phys. Rev. Lett. 95, 195002 (2005).

[9] C. T. M. Chang and J. W. Dawson, J. Appl. Phys. 41, 4493 (1970).

[10] M. Rosing and W. Gai, Phys. Rev. D 42, 1829 (1990).

[11] M.C. Thompson et al., Phys. Rev. Lett. 100, 214801 (2008).

[12] W. Gai, in Proceedings of 2010 International Particle Accelerator Conference (IPAC10), Kyoto, Japan (ICR, Kyoto, 2010), p. 3428.

[13] C. Jing, P. Schoessow, A. Kanareykin, J. G. Power, R. Lindberg, A. Zholents, and P. Piot, in Proceedings of the ICFA Future Light Source Workshop (FLS12), Newport News, VA (2012).

[14] A. Tremaine, J. Rosenzweig, and P. Schoessow, Phys. Rev. E 56, 7204 (1997).

[15] S. Y. Park and J.L. Hirshfield, Phys. Rev. E 62, 1266 (2000).

[16] B. M. Bolotvskii, Usp. Fiz. Nauk 75, 295 (1961) [Sov. Phys. Usp. 4, 781 (1962)].

[17] W. Liu and W. Gai, Phys. Rev. ST Accel. Beams 12, 051301 (2009).

[18] G. V. Sotnikov, T. C. Marshall, and J. L. Hirshfield, Phys. Rev. ST Accel. Beams 12, 061302 (2009).

[19] J. G. Power, W. Gai, and P. Schoessow, Phys. Rev. E 60, 6061 (1999).
[20] W. Gai, R. Konecny, and J. Simpson, in Proceedings of the 2007 Particle Accelerator Conference, Albuquerque, New Mexico (IEEE, New York, 2007), p. 636.

[21] J.M. Fang et al., in Proceedings of the 18th Particle Accelerator Conference, New York, 1999 (IEEE, New York, 1999), p. 3627.

[22] C. Jing, A. Kanareykin, J. G. Power, M. Conde, Z. Yusof, P. Schoessow, and W. Gai, Phys. Rev. Lett. 98, 144801 (2007).

[23] L. Xiao, W. Gai, and X. Sun, Phys. Rev. E 65, 016505 (2001).

[24] C. Jing, W. Liu, L. Xiao, W. Gai, and P. Schoessow, Phys. Rev. E 68, 016502 (2003).

[25] C. Wang and J. L. Hirshfield, Phys. Rev. ST Accel. Beams 9, 031301 (2006).

[26] R. Brinkmann, Y. Derbenev, and K. Flöttmann, Phys. Rev. ST Accel. Beams 4, 053501 (2001).

[27] P. Piot, Y.-E Sun, and K.-J. Kim, Phys. Rev. ST Accel. Beams 9, 031001 (2006).

[28] R. E. Collin, "Field Theory of Guided Waves" (1960).

[29] R. Ruth et al., Part. Accel. 17, 171 (1985); K. Bane et al., IEEE Trans. Nucl. Sci. 32, 3524 (1985).

[30] R. W. Hockney and J. W. Eastwood, Computer Simulation using Particles (Adam Hilger, Bristol and New York, 1988).

[31] K. Bane et al., IEEE Trans. Nucl. Sci. 32, 3524 (1985).

[32] A.W. Chao, Physics of Collective Beam Instabilities in High Energy Accelerators, Wiley Series in Beam Physics \& Accelerator Technology (John Wiley \& Sons, Inc., New York, 1993), ISBN O-471-55 184-8.

[33] S. Vaganian and H. Henke, Part. Accel., 48, 239 (1995).

[34] C. Nieter and J.R. Cary, J. Comput. Phys. 196, 448 (2004); see also http://www.txcorp.com/.

[35] J. Berenger, J. Comput. Phys. 114, 185 (1994).

[36] A. Burov, S. Nagaitsev, and Ya. Derbenev, Phys. Rev. E 66, 016503 (2002).

[37] R. Brinkmann, Ya. Derbenev, and K. Flöttmann, Phys. Rev. ST Accel. Beams 4, 053501 (2001).

[38] Y.-E Sun, P. Piot, K.-J. Kim, N. Barov, S. Lidia, J. Santucci, R. Tikhoplav, and J. Wennerberg, Phys. Rev. ST Accel. Beams 7, 123501 (2004).

[39] K.-J. Kim, Phys. Rev. ST Accel. Beams 6, 104002 (2003).

[40] M. Church, S. Nagaitsev, and P. Piot, in Proceedings of the 2007 Particle Accelerator Conference, Albuquerque, New Mexico (Ref. [20]), p. 2942.

[41] J. Qiang, S. Lidia, R. D. Ryne, and C. Limborg-Deprey, Phys. Rev. ST Accel. Beams 9, 044204 (2006); J. Qiang, R. Ryne, S. Habib, and V. Decyk, J. Comput. Phys. 163, 434 (2000). 